\title{
MAGNETICALLY CONTROLLED INTERFERENCE OF BALLISTIC ELECTRONS TUNNELLED THROUGHOUT THE DOUBLE-BARRIER RESONATOR
}

\author{
T. Figielski, T. Wosiński, A. MąKosa, W. Dobrowolski \\ Institute of Physics, Polish Academy of Sciences \\ Al. Lotników 32/46, 02-668 Warszawa, Poland \\ S.A. Vitusevich and A.E. Belyaev \\ Institute of Semiconductor Physics, National Academy of Sciences of Ukraine \\ 252028 Kiev, Ukraine
}

An effect of magnetic field normal to the tunnel current on the amplitude and phase of the fine oscillatory structure, discovered in the resonance current-voltage curve in double-barrier AlAs/GaAs heterostructures, has been examined. All the obtained results are consistently explained in terms of the interference of ballistic electrons, escaped from the quantum well, in the collector part of the structure.

PACS numbers: 73.40.Gk, 85.30.Mn

Recently, we have discovered fine oscillatory structure (FOS) of the resonant tunnel current flowing through a double-barrier heterostructure (DBII) whose active part consisted of two very thin $(\approx 2 \mathrm{~nm})$ AlAs barriers separated by a GaAs quantum well $(\approx 5 \mathrm{~nm}$ width) $[1,2]$. This FOS displays a surprisingly regular sinusoidal shape with the period in the range $20-40 \mathrm{mV}$. Until this work, the nature of FOS has not been definitely determined.

DBHs which exhibited FOS were grown by molecular beam epitaxy on (001)-oriented GaAs substrates. Square $16 \times 16[\mu \mathrm{m}]^{2}$ mesas were defined on the wafers. The static current-voltage characteristics of each device displayed at least two resonance peaks at both bias polarities, each ending in current bistability.

In this work we have examined the behaviour of FOS in magnetic field applied normally to the tunnel current. Two striking properties of FOS have been found which are discussed in the following:

(i) With increasing magnetic field, the FOS amplitude rapidly falls down to zero, reaching it at $B=1.4 \mathrm{~T}$, but further goes up and falls down again, thus pointing out its oscillatory character (Fig. 1). This is in contrast to the case of 


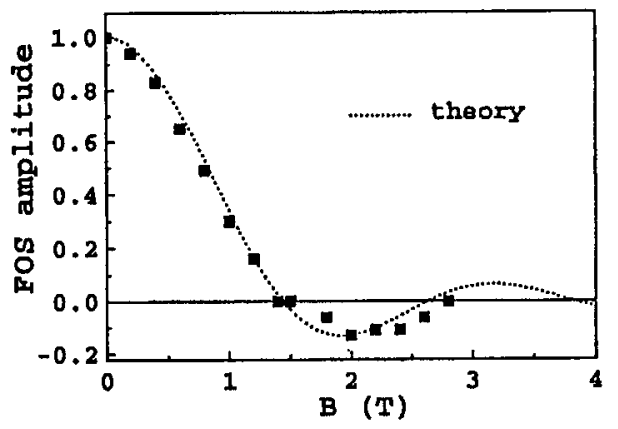

Fig. 1. Relative amplitude of the fine oscillatory structure as a function of magnetic field normal to the current flow, measured at a temperature of $2 \mathrm{~K}$. Dotted line represents theoretical curve, described in the text, fitted to the experimental data.
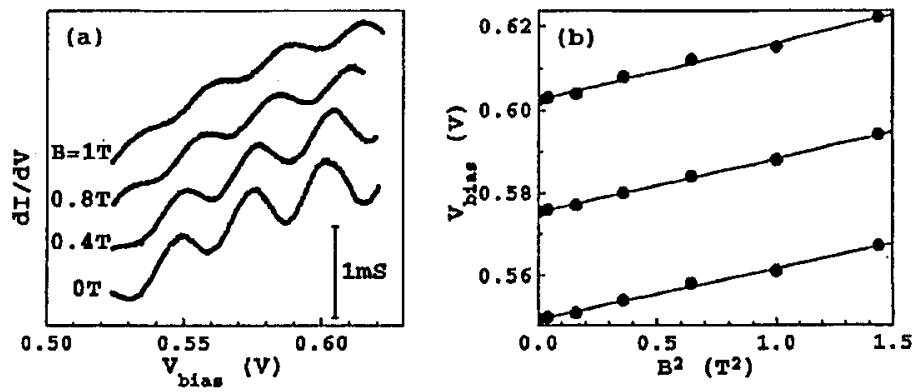

Fig. 2. The effect of magnetic field, normal to the current flow, on the fine oscillatory structure at a temperature of $2 \mathrm{~K}$. (a) Differential conductance vs. bias voltage in different magnetic fields, (b) bias voltages corresponding to the maxima of several cycles of oscillations as a function of the square of magnetic field.

magnetic field applied parallel to the tunnel current, when the FOS amplitude slowly decreases with increasing $B$ but is still observable at $13 \mathrm{~T}$ [2].

(ii) While the positions and shapes of the resonance peaks remain unchanged for $B \leq 2 \mathrm{~T}$, the FOS phase shifts on the bias-voltage scale proportionally to the square of the magnetic field induction (Fig. 2).

These both properties can be consistently explained on the ground of a simple quantitative model which has been proposed [3]. The model assumes that a high-energetic ballistic electron which escaped from the quantum well is partially reflected at some potential step on the collector side of device, situated at a distance $L$ from the back boundary of the collector barrier. Such a step can in principle arise at each interface between two technologically different epilayers. The back-reflected de Broglie wave interferes with the wave running in forward direction (along the $x$-axis) yielding a standing-wave component. Consequently, the square modulus of the wave function between the collector barrier and potential step contains a part oscillating with the distance, $\left|\psi_{\mathrm{osc}}(x)\right|^{2}$. Making a simplified assumption that 
the kinetic energy of an electron outgoing from the well is constant and equal to $U_{\mathrm{b}} \equiv e V_{\text {bias }}$, one gets

$$
\left|\psi_{\text {osc }}(x)\right|^{2} \propto \cos \left(2 k_{x} x\right) \text {. }
$$

For a given $x=L$, this quantity oscillates also with the variation of applied bias. Each minimum of $\left|\psi_{\text {osc }}(L)\right|^{2}$ corresponds to the alignment of self-energies for a "cavity" $[0, L]$ with the energy of the outgoing electron. Therefore, the total transmittance through the considered system oscillates versus bias voltage similarly as $\left|\psi_{\text {osc }}(L)\right|^{2}$ does.

This observation allows us to readily derive an expression for the approximate period of the oscillations, $\Delta U_{\text {osc }}$

$$
\Delta U_{\mathrm{osc}}=\frac{\pi \hbar}{L} \sqrt{\frac{2 U_{\mathrm{b}}}{m}}
$$

where $m$ is the effective mass of the electron, and the distance $L$ is treated here as a free parameter of the model.

We can now discuss the effect of transverse magnetic field (applied along the $z$-axis) on the oscillatory structure. The magnetic field exerts the Lorentz force on the $x$ - and $y$-component of the electron motion. In classical approximation of weak magnetic field, an electron of the wave vector $k=\left[k_{x}, k_{y}, k_{z}\right]$ after travelling a distance $L$ gains under the action of this force an additional momentum along the $x$-axis. It is given by the sum of a linear- and quadratic-versus $B$ terms

$$
\Delta k_{x}=-\frac{k_{y} e B L}{\hbar k_{x}}-\frac{e^{2} B^{2} L^{2}}{2 \hbar^{2} k_{x}} .
$$

The quadratic term is the same for each transmitted electron. It gives rise to a change in the phase of the oscillations which can be expressed in units of the bias voltage as

$$
\Delta U(B)=\frac{e^{2} B^{2} L^{2}}{2 m}
$$

Equation (4) predicts the phase shift proportional to $B^{2}$, just as that observed for FOS.

The linear term in Eq. (3), after integration over all $k_{y}$ available in the electron gas of the emitter, defines the amplitude of the oscillations. If the emission occurs from a two-dimensional electron gas in the accumulation layer of the emitter, the amplitude of the oscillations is expressed as $J_{1}(\alpha B) / \alpha B$. This is an oscillatory function of $B$, which describes well the behaviour of the observed FOS amplitude (dotted line in Fig. 1). Here $J_{1}$ is the Bessel function of the first kind and first order, $\alpha=e L^{2} k_{\mathrm{F}} \sqrt{2 / m U_{\mathrm{b}}}$, and $k_{\mathrm{F}}$ is the Fermi wave vector of the electron gas in the emitter.

One may combine Eqs. (2) and (4) to eliminate uncertain effective mass from these equations to obtain more reliable values for the parameter $L$. All such obtained values for different structures measured up to now and both bias polarities lie within the range $110-190 \mathrm{~nm}$. Usually, the determined values of $L$ coincides quite well with the widths of technologically different epilayers beyond the collector barrier, but sometimes this coincidence is bad.

In the model proposed above we assumed the coherence of wave functions of individual electrons during the resonant tunnelling. It seems that the coherent 
process [4] and not the sequential one [5] dominates the resonant tunnelling in DBHs displaying FOS. This could explain why the fine oscillatory structure is rather uncommon property of the double-barrier heterostructures.

In conclusion, our model of the fine oscillatory structure invoking electron interference effects is self-consistent and explains well the experimental results, but the place where the de Broglie wave of electrons outgoing from the quantum well is reflected, remains discussible.

This work was supported by the Committee for Scientific Research (Poland) under grant No. 2 P03B 035 08, and the State Committee of Science and Technology of Ukraine under grant No. 7.01.05/141.

\section{References}

[1] S.A. Vitusevich, T. Figielski, A. Mąkosa, T. Wosiński, A.E. Belyaev, R.V. Konakova, L.N. Kravchenko, Acta Phys. Pol. A 87, 377 (1995).

[2] T. Figielski, S.A. Vitusevich, A. Mąkosa, W. Dobrowolski, A.E. Belyaev, T. Wosiński, R.V. Konakova, L.N. Kravchenko, Solid State Commun. 94, 93 (1995).

[3] T. Figielski, T. Wosiński, S.A. Vitusevich, A.E. Belyaev, A. Mąkosa, W. Dobrowolski, submitted for publication.

[4] B. Ricco, M.Ya. Azbel, Phys. Rev. B 29, 1970 (1984).

[5] S. Luryi, Appl. Phys. Lett. 47, 490 (1985). 